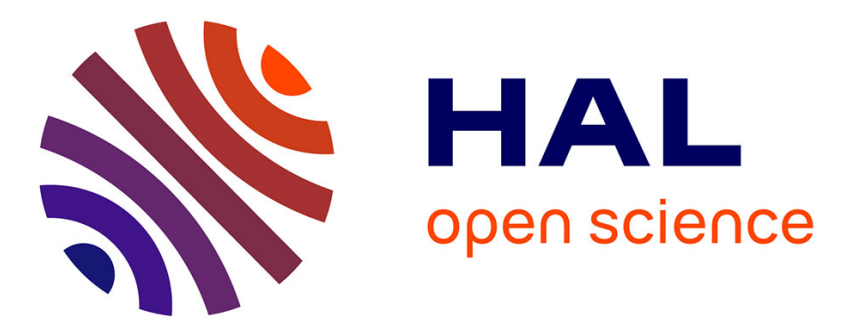

\title{
Protease-activated receptor 2 contributes to Toxoplasma gondii-mediated gut inflammation
}

\author{
Chrystelle Bonnart, Guylène Feuillet, Virginie Vasseur, Nicolas Cénac, \\ Nathalie Vergnolle, N. Blanchard
}

\section{- To cite this version:}

Chrystelle Bonnart, Guylène Feuillet, Virginie Vasseur, Nicolas Cénac, Nathalie Vergnolle, et al.. Protease-activated receptor 2 contributes to Toxoplasma gondii-mediated gut inflammation. Parasite Immunology, 2017, 39 (11), 10.1111/pim.12489 . hal-02624044

\section{HAL Id: hal-02624044 \\ https://hal.inrae.fr/hal-02624044}

Submitted on 26 May 2020

HAL is a multi-disciplinary open access archive for the deposit and dissemination of scientific research documents, whether they are published or not. The documents may come from teaching and research institutions in France or abroad, or from public or private research centers.
L'archive ouverte pluridisciplinaire HAL, est destinée au dépôt et à la diffusion de documents scientifiques de niveau recherche, publiés ou non, émanant des établissements d'enseignement et de recherche français ou étrangers, des laboratoires publics ou privés.

\section{(ㄷ)(1)}

Distributed under a Creative Commons Attribution| 4.0 International License 
DR CHRYSTELLE BONNART (Orcid ID : 0000-0002-3875-492X)

DR NICOLAS BLANCHARD (Orcid ID : 0000-0002-4924-2161)

Article type : Original Paper

\section{Protease-Activated Receptor 2 contributes to Toxoplasma gondii-mediated gut inflammation}

Chrystelle Bonnart ${ }^{1, \$}$, Guylène Feuillet $^{1}$, Virginie Vasseur $^{2}$, Nicolas Cenac ${ }^{1}$, Nathalie Vergnolle $^{1, *}$ and Nicolas Blanchard ${ }^{2, *}$

${ }^{1}$ Institut de Recherche en Santé Digestive (IRSD), Université de Toulouse, INSERM, INRA, ENVT, UPS, Toulouse, France

${ }^{2}$ Centre de Physiopathologie de Toulouse Purpan (CPTP), Université de Toulouse, INSERM, CNRS, UPS, Toulouse, France

*These authors contributed equally to this work

${ }^{\$}$ Corresponding author, address correspondence: chrystelle.bonnart@inserm.fr

Contact information of corresponding author:

INSERM U1220 - IRSD - BatB CHU Purpan BP 302831024 Toulouse Cedex3

This article has been accepted for publication and undergone full peer review but has not been through the copyediting, typesetting, pagination and proofreading process, which may lead to differences between this version and the Version of Record. Please cite this article as doi:

10.1111/pim.12489

This article is protected by copyright. All rights reserved. 
Phone number: +33562744504

Fax number: +33562744558

The experiments were designed by $\mathrm{CB}, \mathrm{NV}$ and $\mathrm{NB}$. $\mathrm{CB}, \mathrm{GF}, \mathrm{VV}$ performed the experiments and analyzed the data. The writing of the manuscript was performed by CB with the help of NV and NB.

Disclosures: None

\section{Abstract}

Toxoplasma gondii is a widespread intracellular parasite, which naturally enters the organism via the oral route and cross the intestinal barrier to disseminate. In addition to neuronal and ocular pathologies, this pathogen also causes gut inflammation in a number of animals. This infection-triggered inflammation has been extensively studied in the C57BL/6 mice, highlighting the importance of the immune cells and their mediators in the development of gut pathology. However, despite their importance in inflammation, the role of Protease-Activated Receptors (PAR) was never reported in the context of T.gondii-mediated small intestine inflammation. Using genetically modified mice, we show that $\mathrm{PAR}_{2}$ plays a pathogenic role in the development of gut inflammatory lesions. We find that $\mathrm{PAR}_{2}$ controls the innate inflammatory mediators IL-6, $\mathrm{KC} / \mathrm{CXCL1}, \mathrm{PGE}_{2}$ as well as neutrophil infiltration in $T$. gondii-triggered gut damage. These results bring new knowledge on the mechanisms operating in the gut in response to $T$. gondii infection.

This article is protected by copyright._All rights reserved. 
Keywords: Toxoplasma gondii, gut infection, inflammation, Protease-Activated Receptor, ileitis

\section{Introduction}

The mucosal barrier constitutes the first line of exchange and defense between our body and the surrounding environment. This natural obstacle has to be crossed in order for a number of harmful enteric pathogens such as Salmonella, but also parasites like Toxoplasma gondii (T. gondii), to penetrate the host. $T$. gondii belongs to the group of Apicomplexa protozoan parasites. T. gondii infection is widespread in humans with dramatic consequences in immunocompromised patients and in fetus of primo-infected pregnant women. Recent growing evidences suggest that $T$. gondii infection might also be a risk factor for developing neuropsychiatric disorders (1).

Apart from neurological and ocular pathologies, another level of interest linked to $T$. gondii invasion in the gut is the observation that a number of mammals and primates display small intestine inflammation upon $T$. gondii infection (2). This pinpoints an acute effect of $T$. gondii infection on gut pathology in animal kingdom. This $T$. gondii-driven small intestine inflammation is observed in the susceptible C57BL/6 (B6) mice. As referred in the literature (3), ingestion of $T$. gondii cysts in B6 mice results in ileitis associated with a massive necrosis causing death of the animals within 10 days.

Previous studies have established a major role for immune cells in the development of intestinal inflammation in this model, including CD4+ T cells (3), Th17 (4), intra-epithelial lymphocytes (5), Natural Killer (NK) cells (6) and innate lymphoid cells (7). Furthermore, the collapse of regulatory T cells together with the subversion of their suppressive capacity is a major event in $T$. gondii-induced intestinal

This article is protected by copyright._All rights reserved. 
pathology (8). $\mathrm{Gr}^{+}$inflammatory monocytes play a protective role but not neutrophils that rather contribute to the intestinal damage $(9,10)$. In addition, the intestinal microflora is believed to play a critical role in the development of gut lesions (11). Regarding the molecular mechanisms, adaptive and innate immunity components have been shown to positively or negatively impact the development of inflammation in this model. Pathogenic functions have been attributed to IFN- $\gamma$, TNF $\alpha$, iNOS $(3,12)$ and TLR9 (13), while IL-10, TGF- $\beta$ and NOD2 appear protective (14-16). However, the role of proteases and their signaling pathways have never been investigated in this pathology.

Proteolytic homeostasis plays a prominent role at mucosal surfaces (17), particularly in the context of intestinal inflammation (18), (19). As a matter of fact, proteases, historically considered as simple digestive enzymes, are now well recognized for their capacity to turn on and off cellular signaling pathways (17). That concept is well illustrated by the ProteaseActivated Receptor (PAR) family (20). PARs are G protein-coupled receptors (GPCRs), which are present at the surface of a large number of cell types. These receptors are activated through proteolytic cleavage of their extracellular $\mathrm{N}$-terminal domain, thereby creating a new $\mathrm{N}$-terminal fragment that serves as a tethered ligand able to bind to the second extracellular loop of the cleaved receptor. This results in signal transduction generally leading to the initiation of a host inflammatory response. PARs are indeed involved in a number of inflammatory or infectious conditions of the oral mucosa and digestive tract $(20,21)$, but their specific role during T. gondiitriggered gut pathology has never been addressed.

Using genetically modified mice, our study demonstrates that $\mathrm{PAR}_{2}$ has a proinflammatory role during T. gondii-triggered small intestine pathology. Moreover, our results indicate that $\mathrm{PAR}_{2}$ is involved in the up-regulation of pro-inflammatory mediators like IL6 and $\mathrm{KC} / \mathrm{CXCL1}$, as well as in the local production of bioactive lipids such as $\mathrm{PGE}_{2}$. This sheds new light on the pathways involved in $T$. gondii-induced intestinal inflammation.

This article is protected by copyright. All rights reserved. 


\section{Materials and Methods}

\section{Mouse model and in vivo infection}

$\mathrm{PAR}_{2}{ }^{-/-}$animals on C57BL/6 (B6) background (22) were compared to either purchased C57BL/6 wild-type mice from Janvier Laboratory or $\mathrm{PAR}_{2}{ }^{+/+}$littermates. Parasite cysts were maintained in CBA/J mice through serial passage of cysts every 8 weeks. Seven week old $\mathrm{PAR}_{2}^{+/+}$and $\mathrm{PAR}_{2}^{-/-}$ mice were orally infected with 30 cysts of $76 \mathrm{~K} T$. gondii strain as described (23). Mice were euthanized at day 7 post-infection. Experimental data results from at least 2 independent experiments using a minimum of $n=7$ animals per group. For the kinetics experiments using WT B6 animals (Fig. 1), a minimum of $n=4$ animals per group was used. Animal studies were carried out under the control of the French National Veterinary Services and in accordance with the European regulations (EEC directive 86/609 dated 24 November 1986). The protocol was approved by the local Ethics Committee CEEA122 (Approval CE n²015-02).

\section{Macroscopic and microscopic scoring of inflammation}

The macroscopic score was based on visual evaluation of small intestinal damage, using criteria adapted from Motta et al. (18). Strictures and presence of blood in the feces could not be used since those two parameters were never observed in the present model. The following criteria were: tissue swelling $(0=$ no swelling, $1=$ minor, $2=$ prominent, $3=$ important, $4=$ major $)$, change in luminal content color $(0=$ no change, $1=$ change $)$ and presence of pus $(0=$ no, $1=$ minor, $2=$ intermediate, $3=$ important) (maximum score: 8). Small intestine tissues were fixed in $4 \%$ formaldehyde for 24 hours and dehydrated in EtOH $70 \%$ before being embedded in paraffin. Three-micron sections were cut, mounted on slides and stained for hematoxylin \& eosin (HE). Histology analysis was used to assess microscopic scores, which combines defects in mucosal architecture (0-3), cellular infiltration (0-3), crypt morphology (normal $=0$, abnormal $=1$ ), diminution of Goblet cell 
number $($ no diminution $=0$, diminution $=1$ ), diminution of Paneth cell $($ no diminution $=0$, diminution =1) and muscle thickening (0-3) (Maximum scores: 12).

\section{QPCR and RT-qPCR analysis}

RNA was extracted from small intestine tissue using Trizol Reagent (Invitrogen). Reverse transcription was performed from $5 \mu \mathrm{g}$ RNA using the Maxima First Strand cDNA Synthesis Kit (Thermo Scientific). QPCR was performed using SYBR Green master mix on LightCycler 480 apparatus (Roche). The graphs represent means of $\Delta \mathrm{Ct}$ between Hprt and target mRNA. Gapdh was also used as a second house-keeping gene to confirm our results (data not shown). Parasite genomic DNA was extracted from mesenteric lymph nodes (MLN) using blood and tissues kit (Qiagen). Parasite burden in MLN was quantified by qPCR of parasite gDNA. The target gene that was chosen was described earlier and the primers allow the amplification of a $162 \mathrm{bp}$ amplicon (24). The absolute number of parasites per mg of tissue was calculated using a standard curve realized with genomic DNA extracted from a known number of $T$. gondii parasites. All primer sequences and GenBank accession numbers are indicated on Table S1.

\section{Immunofluorescence}

Mouse small intestine tissues were prepared as described above in the paragraph "Macroscopic and microscopic scoring of inflammation". After removal of paraffin at $60^{\circ} \mathrm{C}$, slides were boiled 20 minutes in a citrate buffer pH6. After rehydration, slides were incubated in phosphatebuffered saline (PBS) containing 4\% goat serum. Slides were then incubated overnight in the same buffer containing primary antibodies, i.e. rat anti-Ly6G (Biolegend, clone 1A8) or rat IgG2a isotype antibodies (Biolegend). After three washes in PBS, slides were incubated with goat anti-rat Alexa 555 secondary antibodies (Invitrogen), and in a solution of Dapi (500 ng/mL)

This article is protected by copyright. All rights reserved. 
before mounting with Prolong (Invitrogen). Tissues were observed using a Zeiss 710 confocal microscope with a 20x objective.

\section{Quantification of Poly Unsaturated Fatty Acid metabolites}

Analysis was performed by LC-MS/MS as described (25).

\section{Statistical analysis}

All statistical analysis were performed on Graphpad Prism 6 software. Results are expressed as mean \pm SD. Groups were compared using non-parametric tests, i.e. Kruskal-Wallis, Dunn's multiple comparison tests. For neutrophil infiltration in infected animals, Mann-Whitney test was applied. For survival curves, a Mantel-Cox test was used. Unless otherwise stated, experimental data results from at least 2 independent experiments using a minimum of $n=7$ animals per group.

\section{Results}

PAR $_{2}$ mRNA level is increased in inflamed murine small intestine following Toxoplasma gondii infection

Inflammation was induced by oral infection of B6 mice by gavage with 30 cysts of the 76K T. gondii strain. An initial time-course experiment performed at days 3, 5 and 7 postinfection showed a gradual increase in parasite burden quantified by RT-qPCR of SAG1 in small intestine tissues and qPCR on genomic DNA from mesenteric lymph nodes (Fig. 1A,B). This correlated with the progressive setting of small intestine inflammation, as illustrated by increased macroscopic and microscopic scores of inflammation (Fig. 1C,D). Significant increased levels of IFN $\gamma$ and TNF $\alpha$ were observed at day 7 post-infection (Fig. 1E,F). Since sustained $\mathrm{PAR}_{2}$ activation promotes a regulatory feedback loop that augments the level of its own mRNA (26),

This article is protected by copyright. All rights reserved. 
we investigated the impact of $T$. gondii infection on $\mathrm{PAR}_{2}$ transcript level in the small intestine of infected B6 mice. Our results showed that $\mathrm{PAR}_{2}$ mRNA was significantly increased at day 7 post-infection, suggesting a possible involvement of $\mathrm{PAR}_{2}$ in $T$. gondii-driven inflammation (Fig. 1G).

\section{$\mathbf{P A R}_{2}$ deficiency reduces gut inflammation induced by Toxoplasma gondii}

To evaluate the role of $\mathrm{PAR}_{2}$ in the $T$. gondii gut inflammation model, we next infected B6 mice invalidated or not for $\mathrm{PAR}_{2}$ expression. No difference was observed in weight loss between infected WT and KO animals during the week following infection (Fig. 2A). However, higher survival rate was observed in $\mathrm{PAR}_{2}$ deficient infected mice, compared to WT infected mice (Fig 2B). Mice were euthanized at day 7 post-infection to assess gut inflammation. As illustrated in Fig. 3, in infected animals, the macroscopic and microscopic inflammatory scores were significantly decreased in the absence of $\mathrm{PAR}_{2}$ 7-days post infection. This protection was not due to a better control of parasite growth in the gut of $\mathrm{PAR}_{2}$ deficient animals since measurement of $T$. gondii load in the small intestine by RT-qPCR of SAG1 showed similar parasite burden between infected WT and $\mathrm{PAR}_{2}^{-/-}$animals (Fig. 4A). Similar parasite burden was also found in gut draining mesenteric lymph nodes (Fig.4B). Therefore, our results suggest that the development of small intestine inflammation following oral gavage of mice with $T$. gondii cysts involves active $\mathrm{PAR}_{2}$ signaling.

\section{$\mathrm{PAR}_{2}$ is involved in the up-regulation of IL6, KC/CXCL1 cytokines and production of PGE2 and 8-isoPGA2 during Toxoplasma gondii - induced gut pathology}

To decipher which mediators could be regulated by $\mathrm{PAR}_{2}$ during the development of $T$. gondii-mediated gut lesions, we quantified the gene expression level of several inflammatory mediators in the gut tissues of infected and non-infected WT and knockout animals. Gene 
expression of IL-6, KC/CXCL1, CCL2/MCP-1, CCL3/MIP-1A, CCL4/MIP-1B, CCL5/RANTES, NOS2, CXCL2/MIP2, IFN $\gamma$, TNF $\alpha$, IL-10 and TGF- $\beta$ was measured by RTqPCR analysis (Fig. 5). This showed that the amount of IL-6 mRNA level was significantly reduced in infected $\mathrm{PAR}_{2}$-deficient mice compared to WT animals (Fig. 5A). Moreover, the upregulation of KC/CXCL1 level observed upon $T$. gondii infection in WT mice was lost in the absence of $\mathrm{PAR}_{2}$ (Fig. 5B). In contrast, CCL2/MCP-1, CXCL10/IP10, CCL3/MIP-1 $\alpha$, CCL4/MIP-1 $\beta$, CXCL2/MIP2, TNF $\alpha$, IFN $\gamma$ and IL-10 were up-regulated similarly between infected WT and $\mathrm{PAR}_{2}^{-/-}$mice (Fig. 5C-J). Surprisingly, CCL5/RANTES expression was downregulated by $T$. gondii infection in WT animals but up-regulated in $\mathrm{PAR}_{2} \mathrm{KO}$ animals (Fig. 5K). A similar tendency was found for iNOS expression (Fig. 5L). Finally, no difference in TGF- $\beta$ expression was detected among the 4 groups (Fig. 5M). These results indicate that the upregulation of some innate pro-inflammatory cytokines, such as IL-6 and KC/CXCL1, depends at least partially on the presence of $\mathrm{PAR}_{2}$ in this infectious model.

PolyUnsaturated Fatty Acids (PUFA) constitute a source of bioactive lipids playing major roles in innate immunity during infection and inflammation (27). We therefore quantified PUFA metabolites present in the gut during T.gondii infection in WT or $\mathrm{PAR}_{2}$ - deficient mice (Fig. 6). Results illustrated in Fig. 6A,B revealed that in wild-type mice, the amount of $\mathrm{PGE}_{2}$ and 8isoPGA2 were both elevated in the small intestine of $T$. gondii-infected mice in comparison with non-infected animals. The absence of $\mathrm{PAR}_{2}$ completely abolished these effects. In contrast, infection had no impact on the level of other bio-active lipids such as 6KPGF1a, 15d-PGJ2, TxB2 and LTB4 (Fig. 6C-F).

This article is protected by copyright. All rights reserved. 


\section{$\mathbf{P A R}_{2}$ is involved in the infiltration of neutrophils in the small intestine tissue during}

\section{Toxoplasma gondii infection}

Since KC/CXCL1 was reduced in $\mathrm{PAR}_{2}$-deficient mice compared to $\mathrm{WT}$, and given the major neutrophil chemo-attracting role of $\mathrm{KC} / \mathrm{CXCL1}$, we evaluated neutrophil infiltration within the small intestine at day 7 post-infection. For this purpose, immunostaining was performed in tissue cross sections using anti-Ly6G antibodies (Fig. 7A). Total number of neutrophils present on the whole tissue section was reported for WT and $\mathrm{PAR}_{2}$ infected animals, as shown in Fig. 7B. Neutrophil infiltration was lower in $\mathrm{PAR}_{2} \mathrm{KO}$ animals compared to WT. Moreover, in WT tissues, a significant proportion of neutrophils was localized at the top of the villi or in the lumen and was associated with epithelial layer erosion, while the majority of neutrophils counted in $\mathrm{PAR}_{2} \mathrm{KO}$ tissues was detected in the middle or bottom part of the villi.

Altogether, our data showed the implication of $\mathrm{PAR}_{2}$ in the inflammatory phenotype developed in the small intestine infected with $T$. gondii. More specifically, innate immunity mediators IL6, KC/CXCL1 as well as $\mathrm{PGE}_{2}$ and 8-isoPGA 2 are under the control of $\mathrm{PAR}_{2}$ during T.gondii-triggered gut pathology. Moreover, our results suggest that $\mathrm{PAR}_{2}$-dependent mechanisms rely on the recruitment of neutrophils in the small intestine, thereby proposing the implication of this immune cell population in the inflammatory response to $T$. gondii.

\section{Discussion}

The present study sheds light on the role played by $\mathrm{PAR}_{2}$ in the establishment of gut mucosal immune response associated with Toxoplasma gondii pathogenic infection. We here show an alleviated gut inflammatory phenotype in $\mathrm{PAR}_{2} \mathrm{KO}$ mice infected by $T$. gondii, 
associated with a better survival rate. In the absence of parasite load difference between WT and $\mathrm{PAR}_{2} \mathrm{KO}$ infected mice, our results suggest that the increased survival rate observed in PAR KO animals likely results from a lower gut inflammation level compared to WT. However, there was no impact on body weight loss. Although body weight loss is a gross parameter to follow gut inflammation, it does not always correlate perfectly. In particular, $\mathrm{PAR}_{2}$-deficient mice submitted to DSS-colitis protocol do not loose less weight than WT mice despite of a significantly alleviated phenotype as described in Hyun et al.(28).

We showed that among a panel of inflammatory chemokines, cytokines, and lipid mediators, only a few were specifically regulated by $\mathrm{PAR}_{2}$ during $T$.gondii infection. We found that expression of IL-6 and KC/CXCL1 cytokines as well as $\mathrm{PGE}_{2}$ and 8-isoPGA 2 lipid production were lower in infected $\mathrm{PAR}_{2}$-deficient animals, compared to WT. In line with the control of $\mathrm{KC} / \mathrm{CXCL1}$ by $\mathrm{PAR}_{2}$ and in agreement with the strong neutrophil chemo-attracting activity of this chemokine, we found that neutrophil infiltration of the tissue in infected animals was significantly reduced in the absence of $\mathrm{PAR}_{2}$. Our results suggest that the mechanisms behind the pathogenic role of $\mathrm{PAR}_{2}$ rely on the control by $\mathrm{PAR}_{2}$ of a specific subset of innate immunity mediators. Our results propose that $\mathrm{PAR}_{2}$-dependent neutrophil infiltration would be due to KC/CXCL1 chemokine up-regulation and that this could constitute a major pathway in the establishment of intestinal lesions during $T$. gondii infection of the gut.

Our manuscript supports the idea of parallel $\mathrm{PAR}_{2}$-dependent and independent mechanisms to control inflammatory mediators and inflammation level in $T$. gondii-mediated gut inflammation model. The fact that inflammation in the gut was not fully abolished in the absence of $\mathrm{PAR}_{2}$ suggests that a part of this inflammation is regulated by $\mathrm{PAR}_{2}$-independent mechanisms that are still in place during infection. This is likely reflected by the fact that the levels of IFN $\gamma$ 
and $\mathrm{TNF} \alpha$, which are described as important mediators in this inflammation model, are upregulated similarly in both groups of infected animals, regardless of $\mathrm{PAR}_{2}$ presence. Interestingly, a lack of correlation between the level of these two major cytokines and the level of gut inflammation has been reported in the literature. This is exemplified in the paper of Guiton et al. (4) where the same T. gondii-mediated ileitis model was used. The authors showed a decrease of gut inflammation in the absence of IL-17 receptor but this was not correlated with decreased levels of IFN $\gamma$ and TNF $\alpha$, which were similarly induced in WT and IL17RA KO infected mice. In addition, akin to our situation, there was a similar parasite burden in the small intestine of both animals. Even more surprising, Bonfa et al. (2014) showed an inverse correlated phenotype where T.gondii-infected CCR5 knockout mice displayed increased gut inflammatory lesions but decreased levels of mRNA levels IFN $\gamma$ and TNF $\alpha$ (and many more cytokines) in the ileum (29). These studies including ours, underline the complexity of mechanisms controlling gut inflammation during $T$. gondii infection and indicate that pathways, unrelated to IFN $\gamma$ and TNF $\alpha$, operate during infection and control the intensity of inflammatory gut lesions.

Several non-exclusive sources of proteases are potentially able to activate $\mathrm{PAR}_{2}$ during $T$. gondii-triggered gut pathology (17). Firstly, proteases with known $\mathrm{PAR}_{2}$-modulating activities are brought by pancreatic secretion (trypsin), or produced in situ by intestinal epithelial cells and/or inflammatory cells (kallikreins, cathepsin S, neutrophil elastase, cathepsin G and proteinase 3)(30-32). Although host cells are considered as the main sources of $\mathrm{PAR}_{2}$-activating proteases, a number of studies show that microbial proteases also participate to the modulation of PAR activity $(33,34)$ and it is logical to speculate that serine proteases from the microbiota as well as T.gondii itself may also cleave the receptor. Although the number of $T$. gondii parasites is low at the initial invasion step, the parasite burden in the small intestine tissue is increasing 
substantially during inflammation progression as a consequence of intra-vacuolar replication in infected cells and neutrophil-mediated spreading throughout the small intestinal lumen (35). T. gondii contains intra-vesicular pools of proteases that are secreted during the invasion process (36) and which remain at the vicinity of membrane receptors during invasion. It is thus conceivable that some of these parasitic proteases from the parasite-secreted microneme vesicles, may directly activate $\mathrm{PAR}_{2}$ and thereby participate to the switch of the intestine towards a proinflammatory phenotype. Among those potential candidate proteases are MPP2, MPP3, TgSUB1, TgROM1 and Rhomboid-like protease 5. Future work will be needed to identify the precise nature of these $\mathrm{PAR}_{2}$-activating proteases and their relevance in the inflammatory response.

Our work demonstrates for the first time the contribution of $\mathrm{PAR}_{2}$ in gut inflammatory responses mediated by $T$. gondii infection. Our study favors a model in which $\mathrm{PAR}_{2}$ controls the level of inflammation and neutrophil infiltration by regulating the level of KC/CXCL1 in the small intestine tissue during $T$. gondii infection. These results highlight the importance of proteolytic homeostasis in the control of the host response to gut infection. Our findings may be relevant for other gut protozoan parasites such as Cryptosporidium, which also belong to Apicomplexa, targets the small intestine and is the causal agent of severe diarrheal pathology worldwide.

This article is protected by copyright.All rights reserved. 


\section{Acknowledgments}

Histology experiments were performed at the Platform of Experimental Histopathology of the INSERM / UPS US006 CREFRE), Toulouse Purpan, France. PAR $_{2}^{---}$and littermates wild type animals were maintained at the animal facility platform of INSERM / UPS US006 CREFRE. We are grateful to Dr Buzoni-Gatel for her kind gift of a 76K-infected mouse. We thank Olivier Joffre (CPTP) for the gift of some qPCR primers. We thank Emilie Bassot (CPTP) for technical assistance with maintenance of $76 \mathrm{~K}$ cysts.

\section{Funding}

This work was supported by Toulouse University emerging project program, the 'Agence Nationale de la Recherche' [ANR-11-BSV3-01002 to N.B. and ANR-12-BSV1-0030-01 to N.V.], the Human Frontier Science Program Organization [CDA00047/2011 to N.B.], the PIA Parafrap Consortium [ANR-11-LABX0024 to N.B.] and PIA ANINFIMIP equipment [ANR-11EQPX-0003 to N.B. and N.V.], and by the European Research Council [ERC-2012-StG20111109 to N.V.].

\section{References}

1. Fabiani S, Pinto B and Bruschi F. Toxoplasmosis and neuropsychiatric diseases: can serological studies establish a clear relationship? Neurological sciences : official journal of the Italian Neurological Society and of the Italian Society of Clinical Neurophysiology 2013; 34: 417-425.

2. Schreiner $M$ and Liesenfeld $\mathbf{O}$. Small intestinal inflammation following oral infection with Toxoplasma gondii does not occur exclusively in C57BL/6 mice: review of 70 reports from the literature. Mem Inst Oswaldo Cruz 2009; 104: 221-233.

3. Liesenfeld 0 , Kosek J, Remington JS and Suzuki Y. Association of CD4+ T cell-dependent, interferon-gamma-mediated necrosis of the small intestine with genetic susceptibility of mice to peroral infection with Toxoplasma gondii. J Exp Med 1996; 184: 597-607.

4. Guiton R, Vasseur V, Charron S et al. Interleukin 17 receptor signaling is deleterious during Toxoplasma gondii infection in susceptible BL6 mice. J Infect Dis; 202: 427-435.

This article is protected by copyright. All rights reserved. 
5. Egan CE, Maurer KJ, Cohen SB, Mack M, Simpson KW and Denkers EY. Synergy between intraepithelial lymphocytes and lamina propria $T$ cells drives intestinal inflammation during infection. Mucosal Immunol 2011; 4: 658-670.

6. Ronet C, Darche S, Leite de Moraes M et al. NKT cells are critical for the initiation of an inflammatory bowel response against Toxoplasma gondii. J Immunol 2005; 175: 899-908.

7. Schulthess J, Meresse B, Ramiro-Puig E et al. Interleukin-15-Dependent NKp46(+) Innate Lymphoid Cells Control Intestinal Inflammation by Recruiting Inflammatory Monocytes. Immunity 2012; 37: 108-121.

8. Oldenhove G, Bouladoux N, Wohlfert EA et al. Decrease of Foxp3+ Treg cell number and acquisition of effector cell phenotype during lethal infection. Immunity 2009; 31: 772-786.

9. Dunay IR, Damatta RA, Fux B et al. Gr1(+) inflammatory monocytes are required for mucosal resistance to the pathogen Toxoplasma gondii. Immunity 2008; 29: 306-317.

10. Dunay IR, Fuchs A and Sibley LD. Inflammatory monocytes but not neutrophils are necessary to control infection with Toxoplasma gondii in mice. Infect Immun 2010; 78: 1564-1570.

11. Heimesaat $\mathrm{MM}$, Bereswill S, Fischer A et al. Gram-negative bacteria aggravate murine small intestinal Th1-type immunopathology following oral infection with Toxoplasma gondii. J Immunol 2006; 177: 8785-8795.

12. Liesenfeld O, Kang H, Park D et al. TNF-alpha, nitric oxide and IFN-gamma are all critical for development of necrosis in the small intestine and early mortality in genetically susceptible mice infected perorally with Toxoplasma gondii. Parasite immunology 1999; 21: 365-376.

13. Minns LA, Menard LC, Foureau DM et al. TLR9 is required for the gut-associated lymphoid tissue response following oral infection of Toxoplasma gondii. J Immunol 2006; 176: 75897597.

14. Suzuki Y, Sher A, Yap G et al. IL-10 is required for prevention of necrosis in the small intestine and mortality in both genetically resistant BALB/C and susceptible C57BL/6 mice following peroral infection with Toxoplasma gondii. J Immunol 2000; 164: 5375-5382.

15. Buzoni-Gatel D, Debbabi H, Mennechet FJ et al. Murine ileitis after intracellular parasite infection is controlled by TGF-beta-producing intraepithelial lymphocytes. Gastroenterology 2001; 120: 914-924.

16. Heimesaat $M M$, Dunay IR, Alutis $M$ et al. Nucleotide-Oligomerization-Domain-2 Affects Commensal Gut Microbiota Composition and Intracerebral Immunopathology in Acute Toxoplasma gondii Induced Murine Ileitis. PloS one 2014; 9: e105120.

17. Vergnolle N. Protease inhibition as new therapeutic strategy for GI diseases. Gut 2016.

18. Motta JP, Bermudez-Humaran LG, Deraison C et al. Food-grade bacteria expressing elafin protect against inflammation and restore colon homeostasis. Science translational medicine 2012; 4: 158ra144.

19. Motta JP, Magne L, Descamps D et al. Modifying the protease, antiprotease pattern by elafin overexpression protects mice from colitis. Gastroenterology; 140: 1272-1282.

20. Vergnolle N. Proteinase-activated receptors (PARs) in infection and inflammation in the gut. The international journal of biochemistry \& cell biology 2008; 40: 1219-1227.

21. Hansen KK, Sherman PM, Cellars L et al. A major role for proteolytic activity and proteinaseactivated receptor-2 in the pathogenesis of infectious colitis. Proc Natl Acad Sci U S A 2005; 102: 8363-8368.

22. Lindner JR, Kahn ML, Coughlin SR et al. Delayed onset of inflammation in protease-activated receptor-2-deficient mice. J Immunol 2000; 165: 6504-6510.

23. Foureau DM, Mielcarz DW, Menard LC et al. TLR9-dependent induction of intestinal alphadefensins by Toxoplasma gondii. J Immunol 2010; 184: 7022-7029. 
24. Reischl U, Bretagne S, Kruger D, Ernault P and Costa JM. Comparison of two DNA targets for the diagnosis of Toxoplasmosis by real-time PCR using fluorescence resonance energy transfer hybridization probes. BMC infectious diseases 2003; 3: 7.

25. Le Faouder P, Baillif V, Spreadbury I et al. LC-MS/MS method for rapid and concomitant quantification of pro-inflammatory and pro-resolving polyunsaturated fatty acid metabolites. Journal of chromatography B, Analytical technologies in the biomedical and life sciences 2013; 932: 123-133.

26. Suen JY, Gardiner B, Grimmond S and Fairlie DP. Profiling gene expression induced by protease-activated receptor 2 (PAR2) activation in human kidney cells. PloS one 2010; 5: e13809.

27. Dennis EA and Norris PC. Eicosanoid storm in infection and inflammation. Nature reviews Immunology 2015; 15: 511-523.

28. Hyun E, Andrade-Gordon $P$, Steinhoff $M$ and Vergnolle N. Protease-activated receptor-2 activation: a major actor in intestinal inflammation. Gut 2008; 57: 1222-1229.

29. Bonfa G, Benevides L, Souza Mdo C et al. CCR5 controls immune and metabolic functions during Toxoplasma gondii infection. PloS one 2014; 9: e104736.

30. Zhao $\mathrm{P}$, Lieu $\mathrm{T}$, Barlow $\mathbf{N}$ et al. Cathepsin $\mathrm{S}$ causes inflammatory pain via biased agonism of PAR2 and TRPV4. J Biol Chem 2014.

31. Ramachandran $\mathrm{R}$, Eissa $\mathrm{A}$, Mihara $\mathrm{K}$ et al. Proteinase-activated receptors (PARs): differential signalling by kallikrein-related peptidases KLK8 and KLK14. Biological chemistry 2012; 393: 421-427.

32. Ramachandran $\mathrm{R}$, Mihara $\mathrm{K}$, Chung $\mathrm{H}$ et al. Neutrophil elastase acts as a biased agonist for proteinase-activated receptor-2 (PAR2). J Biol Chem 2011; 286: 24638-24648.

33. Dulon S, Leduc D, Cottrell GS et al. Pseudomonas aeruginosa elastase disables proteinaseactivated receptor 2 in respiratory epithelial cells. Am J Respir Cell Mol Biol 2005; 32: 411-419.

34. Lourbakos A, Yuan YP, Jenkins $A L$ et al. Activation of protease-activated receptors by gingipains from Porphyromonas gingivalis leads to platelet aggregation: a new trait in microbial pathogenicity. Blood 2001; 97: 3790-3797.

35. Coombes JL, Charsar BA, Han SJ et al. Motile invaded neutrophils in the small intestine of Toxoplasma gondii-infected mice reveal a potential mechanism for parasite spread. Proc NatI Acad Sci U S A 2013; 110: E1913-1922.

36. Carruthers VB. Proteolysis and Toxoplasma invasion. Int J Parasitol 2006; 36: 595-600. 


\section{Figure Legends}

Figure 1. Kinetics of parasite load, inflammatory parameters and $P A R_{2}$ expression in wildtype B6 mice at days 3, 5 and 7 post-infection. A) Parasite burden quantified in the small intestine of B6 mice by RT-qPCR of SAG1 at days 3, 5 and 7 post-infection. Murine HPRT was used as a house keeping gene for normalization. B) Absolute parasite burden quantified in mesenteric lymph nodes by qPCR of $T$. gondii genomic DNA at days 3, 5 and 7 post-infection. C) and D) Macroscopic and microscopic scores of inflammation in the small intestine during $T$. gondii infection. E-F) Quantitative RT-PCR showing the relative expression of IFN $\gamma$ (E) and TNF $\alpha(\mathbf{F})$ in the small intestine tissue. Panels A-F are representative of one experiment using $\mathrm{n}=4$ infected mice for each time point and $\mathrm{n}=3$ to 12 mice for the non infected group. G) RTqPCR of small intestine tissue showing increased expression level of $\mathrm{PAR}_{2}$ mRNA (relative to HPRT) at day 7 post-infection. This figure represents two experiments. NI: Non Infected ; $T$. gondii: T. gondii - infected animals. Statistics were done to compare infected groups with the non infected control group. Only significant differences to the non infected group are indicated. p-value $*<0,05 ; * *<0,01$

Figure 2. Body weight loss and survival curves of WT and PAR $\mathrm{KO}$ mice infected with Toxoplasma gondii. A) The body weight of non infected or T. gondii-infected animals was measured during the week following infection. The graph shows the variation of weight compared to day 0 (corresponding to $100 \%$ ). WT and $\mathrm{PAR}_{2} \mathrm{KO}$ infected animals showed no difference in weight loss (Kruskal-Wallis test). B) Survival curves of T. gondii-infected WT and $\mathrm{PAR}_{2}$ deficient mice showing a better survival in $\mathrm{KO}$ animals compared to WT (statistical

This article is protected by copyright. All rights reserved. 
analysis using Mantel-Cox test). This figure represents one experiment. p-value $*<0,05 ;$ n.s : not significant.

Figure 3. Inflammatory scores in the small intestine of $\mathrm{WT}$ and $\mathrm{PAR}_{2} \mathrm{KO}$ animals infected with Toxoplasma gondii . A) Macroscopic scores of inflammation in the small intestine of animals harvested 7 days post-infection. B) H\&E staining of the small intestine of animals harvested 7 days post-infection. Scale bar: $200 \mu \mathrm{m}$. Microscopic scores of inflammation were based on H\&E picture examination. C-D) Magnification of H\&E pictures. (C) Top of the villi showing erosion of the epithelium layer with decreased in the number of Goblet cells (arrow) and strong immune cell infiltration (numerous small nuclei) in WT infected animals. $\mathrm{PAR}_{2}{ }^{-1-}$ infected animals showed less severe erosion, and some goblet cells were still visible. (D) Bottom of the crypt showing the presence of Paneth cells (red vesicles - containing cells) in non infected WT and $\mathrm{PAR}_{2} \mathrm{KO}$ mice. Upon infection, strong reduction of Paneth cell was observed in WT animals whereas some Paneth cell were still present in $\mathrm{PAR}_{2}$ deficient animals Scale bar: $50 \mu \mathrm{m}$, objective 40X. $\mathrm{n}=3$ independent experiments. NI: Non Infected ; T. gondii : T. gondii - infected animals.

Figure 4. Parasite burden in WT and $\mathrm{PAR}_{2} \mathrm{KO}$ infected mice. A) Parasite burden quantification in WT and $\mathrm{PAR}_{2}^{-{ }^{--}}$small intestine tissues at day 7 post-infection. RT-qPCR analysis showed similar mRNA expression level of SAG1 (major surface antigen of tachyzoite) relative to murine HPRT. No expression of SAG1 was detectable in non infected animals. B) Parasite burden quantified in mesenteric lymph nodes by QPCR at day 7 post-infection, showing similar load between WT and $\mathrm{PAR}_{2}$ mice.

This article is protected by copyright. All rights reserved. 
Figure 5. RT-qPCR quantification of inflammatory mediators in the small intestine of WT and PAR $_{2}$ KO animals infected with Toxoplasma gondii. Quantitative RT-PCR at day 7 postinfection showing the relative expression of IL6 (A), KC/CXCL1 (B), CCL2 (C), CXCL10 (D), CCL3 (E), CCL4 (F), CXCL2 (G), TNF $\alpha(\mathbf{H}), \operatorname{IFN} \gamma(\mathbf{I})$, IL10 (J), CCL5 (K), iNOS (L) and TGF-b (M) in WT or $\mathrm{PAR}_{2}^{-{ }^{--}}$murine small intestine tissue infected or not with T. gondii. The graph represents 2 experiments for IL6, KC/CXCL1, IFN $\gamma$ and TNF $\alpha$ analysis, and 1 experiment for the other mediators. NI: Non Infected ; T. gondii : T. gondii - infected animals. p-value ${ }^{*}<$ 0,$05 ; * *<0,01 ; * * *<0,001 ; * * * *<0,0001 ;$ n.s : not significant.

Figure 6. Quantification of lipid metabolites in the small intestine of WT and $\mathrm{PAR}_{2} \mathrm{KO}$ animals infected with Toxoplasma gondii. Quantification of lipid metabolites in WT or $\mathrm{PAR}_{2}^{-{ }^{-1}}$ small intestine tissues at day 7 post-infection : PGE2 (A), 8-isoPGA2 (B), 6KPGF1a (C), 15dPGJ2 (D), TxB2 (E) and LTB4 (F). NI: Non Infected ; T. gondii : T. gondii - infected animals. The graph results from 2 pooled experiments. p-value $*<0,05 ; * *<0,01 ; * * *<0,001 ;$ n.s : not significant.

Figure 7. Neutrophil infiltration in the small intestine of infected WT and $P_{A} R_{2} K O$ animals at day 7 post-infection. (A) Immunostaining was performed on tissue cross sections using anti-Ly6G antibodies. Pictures show the presence of neutrophils (Ly6G staining in red) associated with areas of tissue erosions in the small intestine from WT infected animals. Tissues from $\mathrm{PAR}_{2} \mathrm{KO}$ showed few Ly6G positive cells, which were restricted to the lamina propria compartment. Scale bar: $100 \mu \mathrm{m}$. DAPI: blue staining. B) Quantification of neutrophils present on tissue cross sections in WT and $\mathrm{PAR}_{2}^{-{ }^{-/}}$infected animals. The total number of Ly6G positive

This article is protected by copyright._All rights reserved. 
cells per cross section of WT or $\mathrm{PAR}_{2}^{-/-}$was reported and showed diminished neutrophil infiltration in $\mathrm{PAR}_{2} \mathrm{KO}$ animals compared to WT. This figure represents one experiment. pvalue $*<0,05$.

This article is protected by copyright. All rights reserved.

Bonnart, C., Feuillet, G., Vasseur, V., Cénac, N., Vergnolle, N., Blanchard, N. (2017) 
Figure 1 Bonnart et al.

A

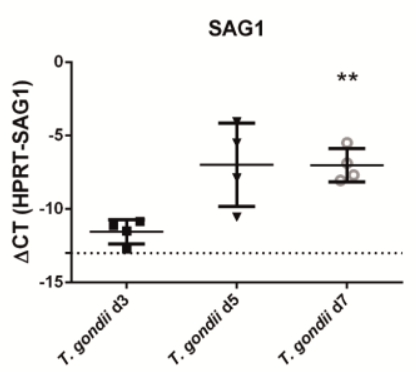

D

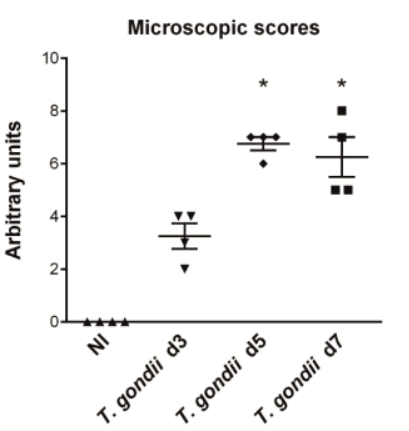

G

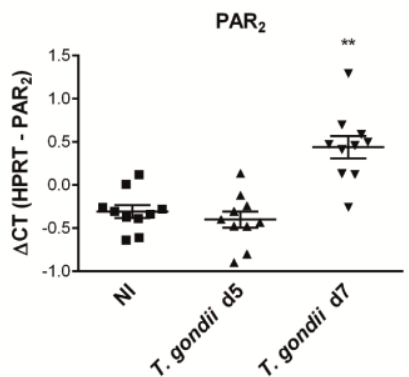

B

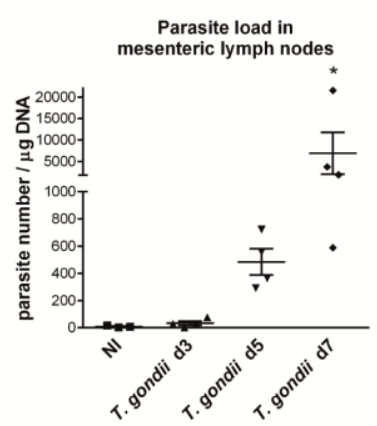

$\mathbf{E}$

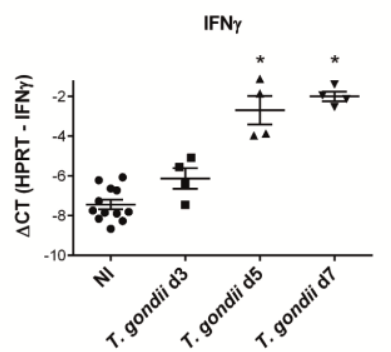

C

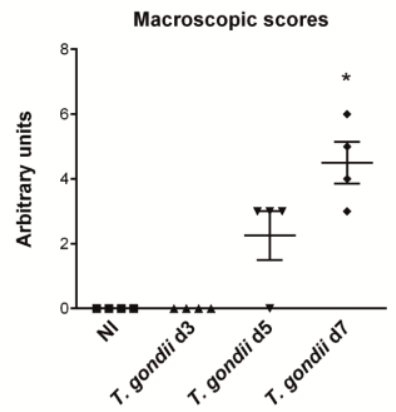

$\mathbf{F}$

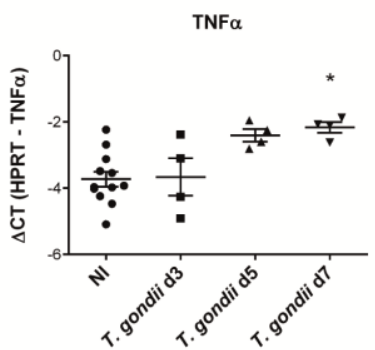

Figure 1. Kinetics of parasite load, inflammatory parameters and PAR 2 expression in wild-type B6 mice at days 3,5 and 7 post-infection.

This article is protected by copyright. All rights reserved. 
Figure 2 Bonnart et al.

A

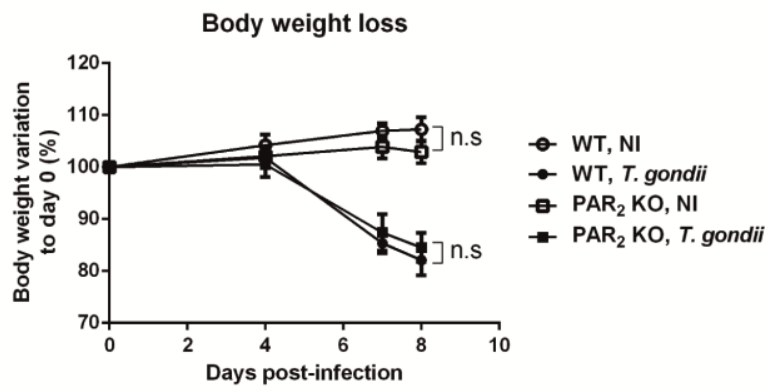

B

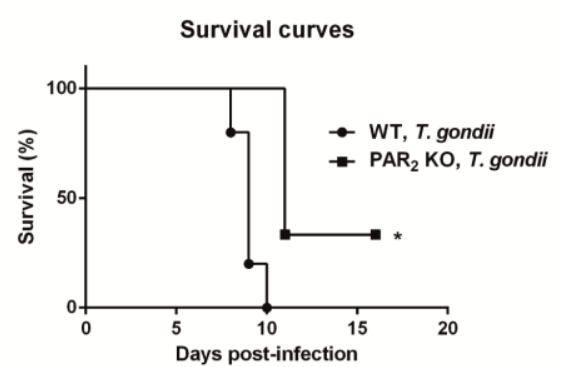

Figure 2. Body weight loss and survival curves of WT and PAR 2 KO mice infected with Toxoplasma gondii.

This article is protected by copyright. All rights reserved.

Bonnart, C., Feuillet, G., Vasseur, V., Cénac, N., Vergnolle, N., Blanchard, N. (2017). 
Figure 3 (A-B) Bonnart et al.

A
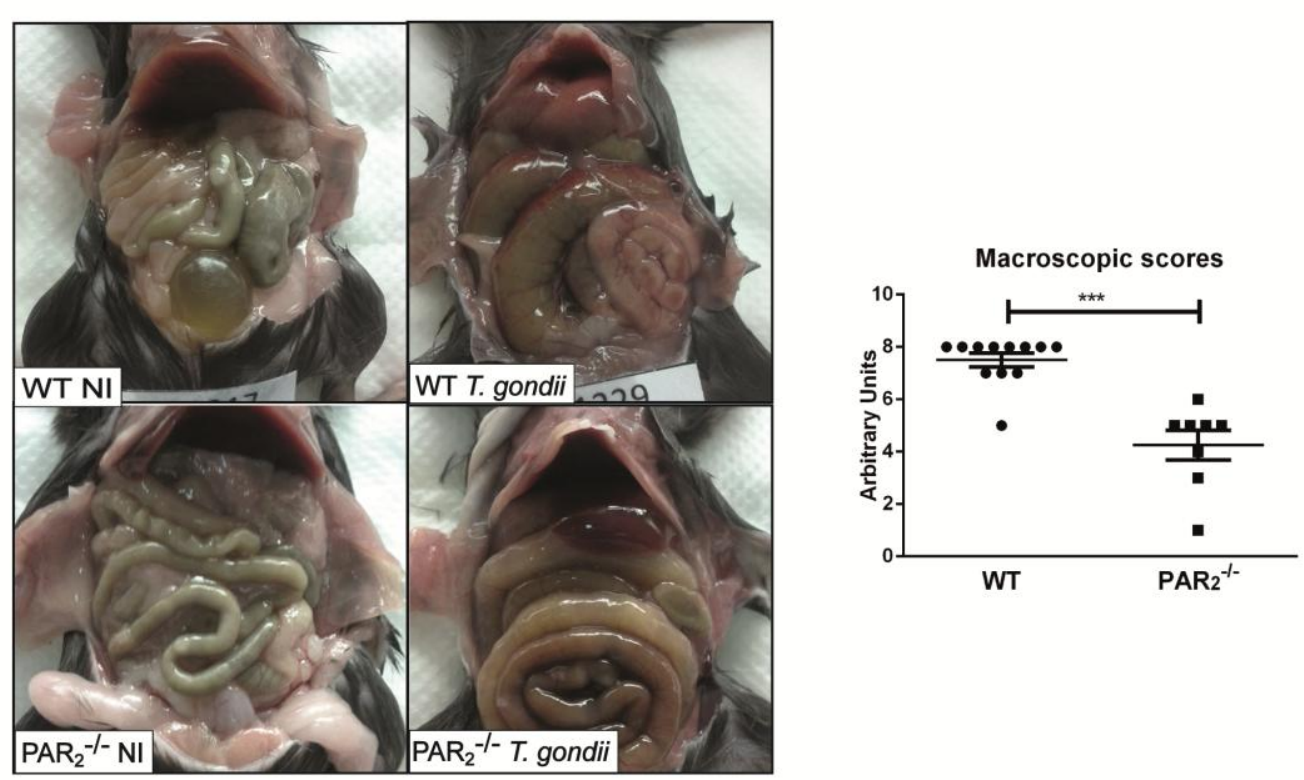

B
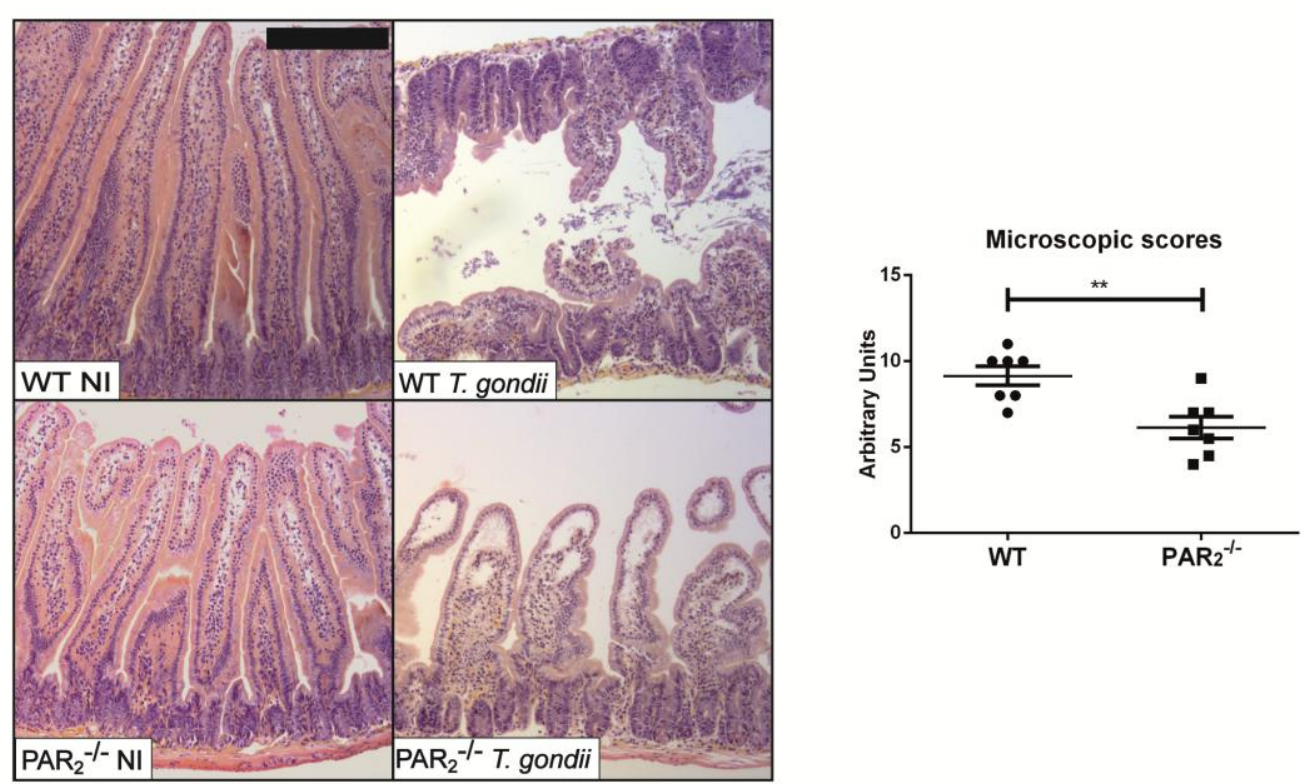

This article is protected by copyright. All rights reserved. 
Figure 3 (C-D) Bonnart et al.
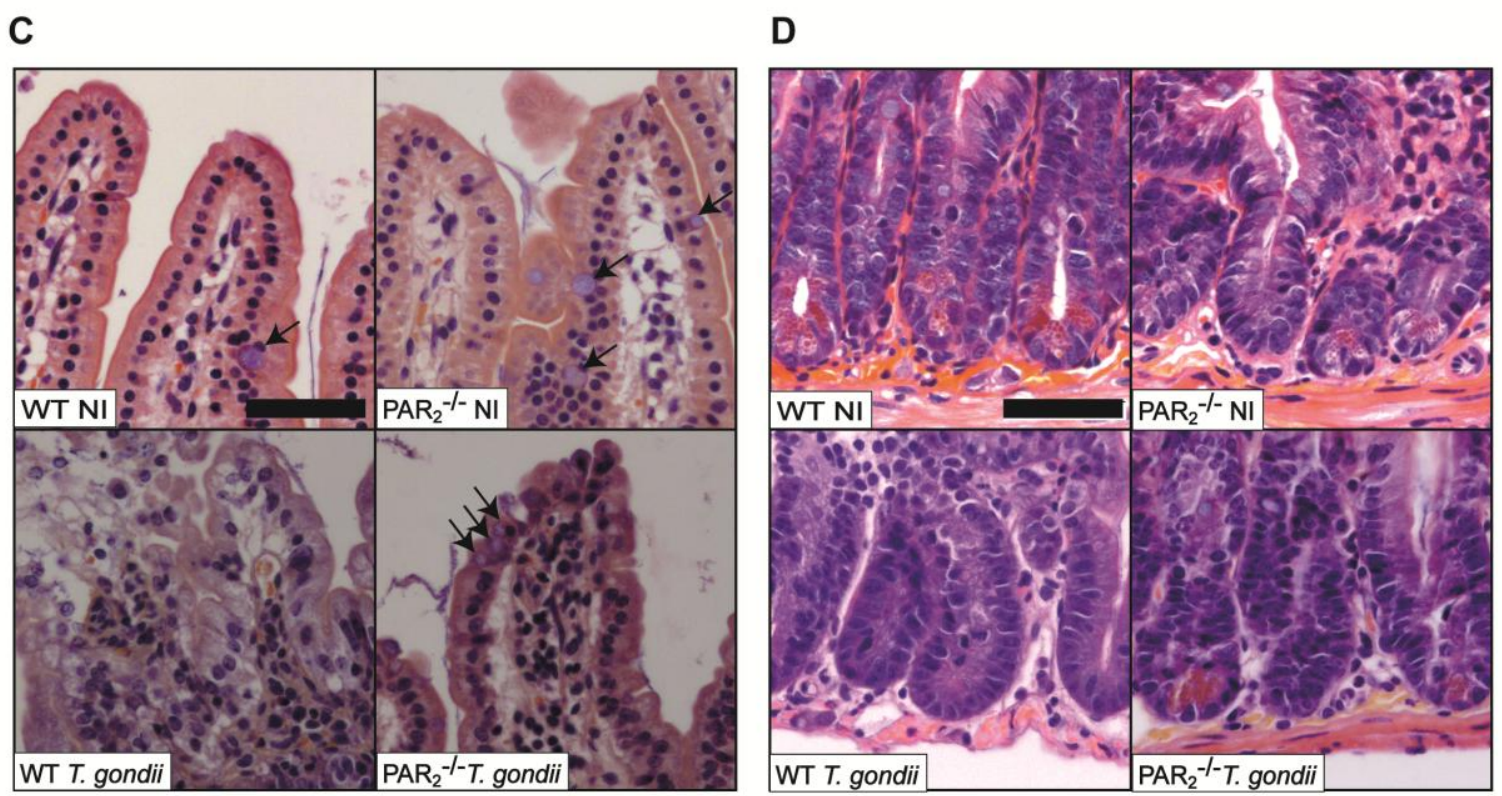

Figure 3. Inflammatory scores in the small intestine of WT and PAR 2 KO animals infected with Toxoplasma gondii.

This article is protected by copyright. All rights reserved.

Bonnart, C., Feuillet, G., Vasseur, V., Cénac, N., Vergnolle, N., Blanchard, N. (2017) 
Figure 4 Bonnart et al.

A

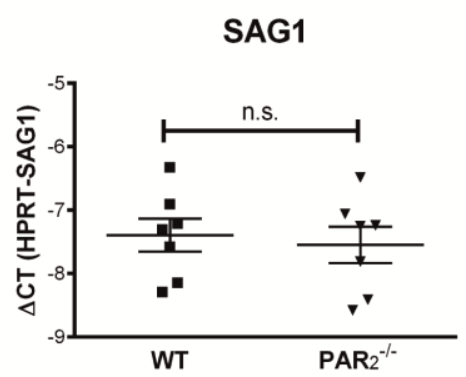

B

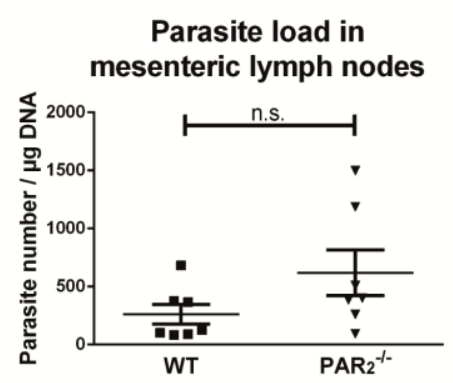

Figure 4. Parasite burden in WT and PAR 2 KO infected mice.

This article is protected by copyright. All rights reserved. 
Figure 5 Bonnart et al.

A

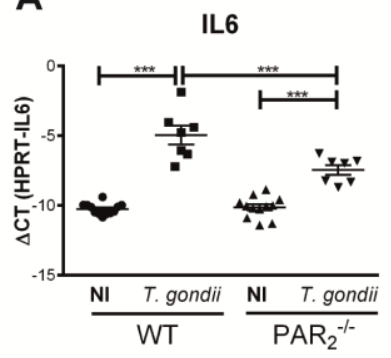

D

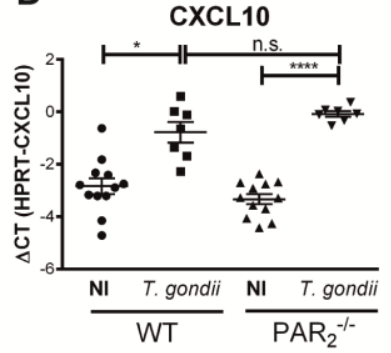

G

CXCL2

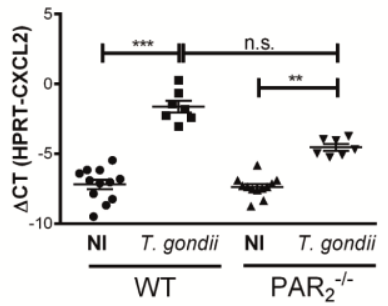

J

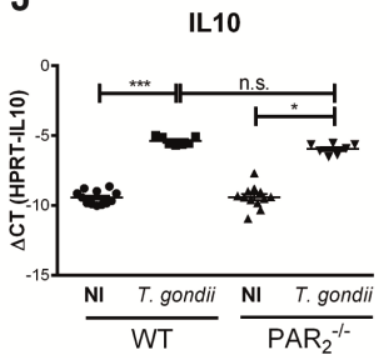

M

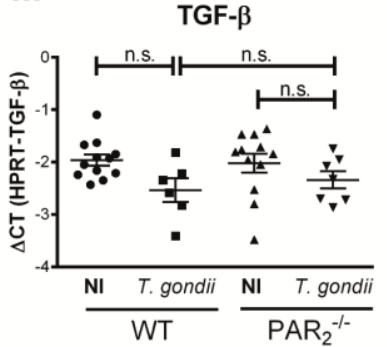

B

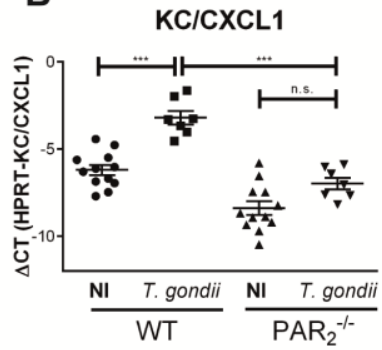

E

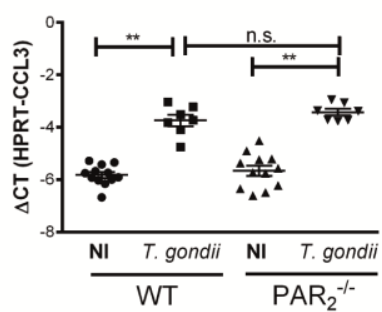

H

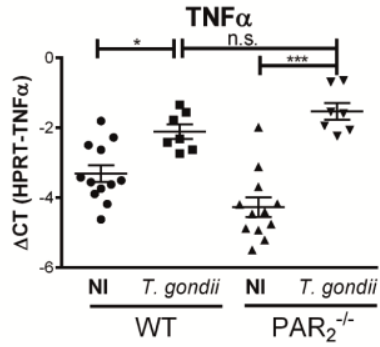

K

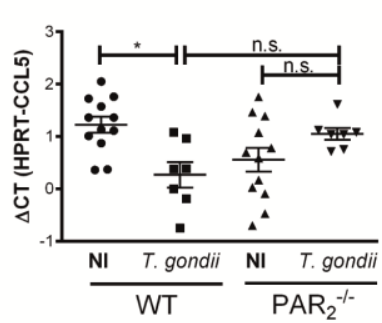

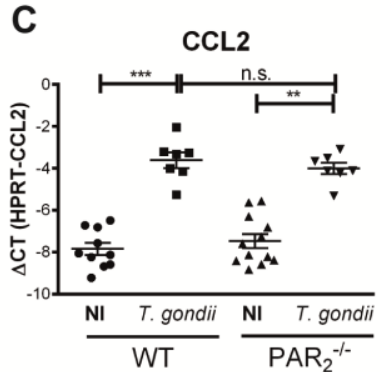

$\mathbf{F}$

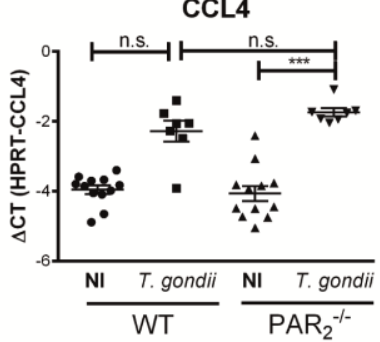

I

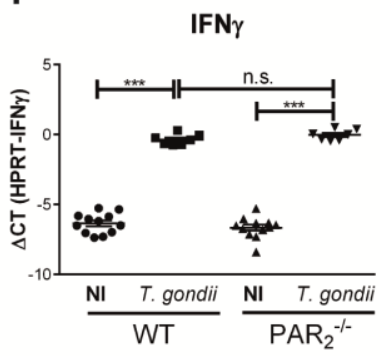

$\mathbf{L}$

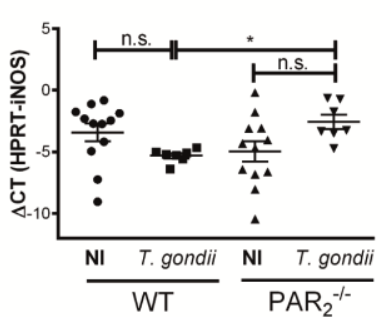

Figure 5. RT-qPCR quantification of inflammatory mediators in the small intestine of WT and PAR2 KO animals infected with Toxoplasma gondii.

This article is protected by copyright. All rights reserved. 
Figure 6 Bonnart et al.

A

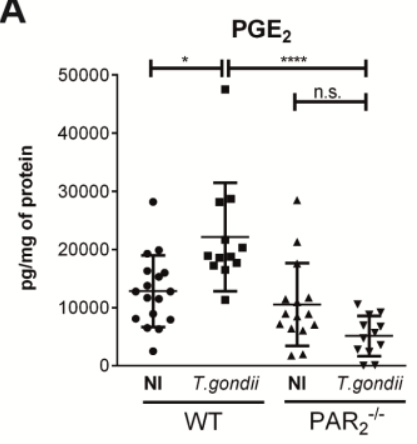

C

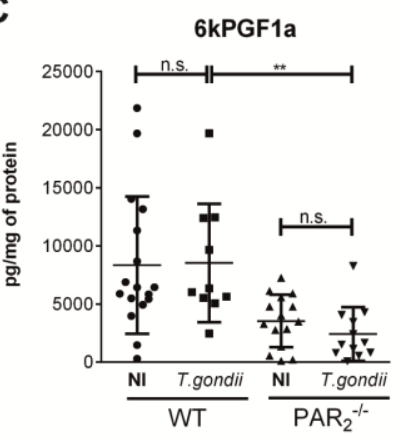

$\mathbf{E}$

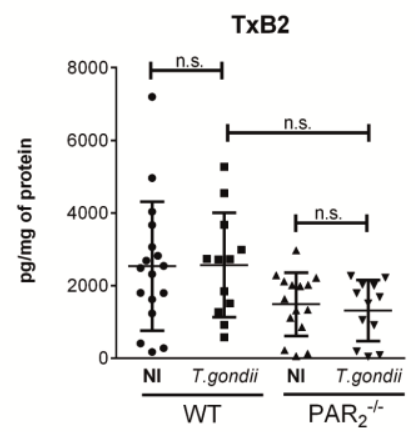

B

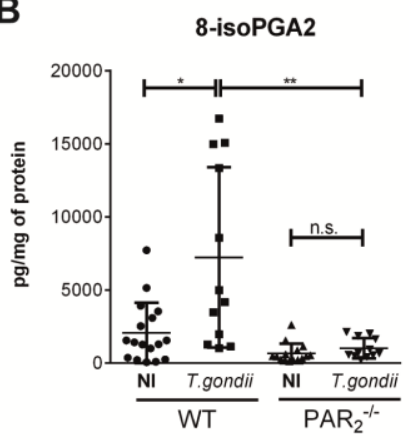

D

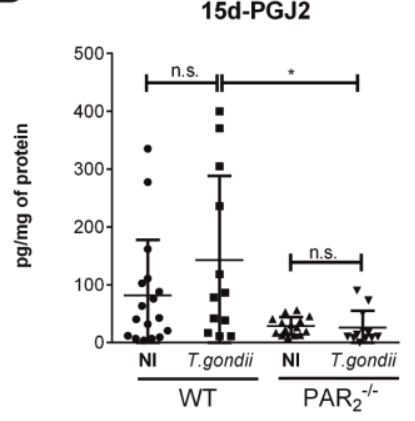

$\mathbf{F}$

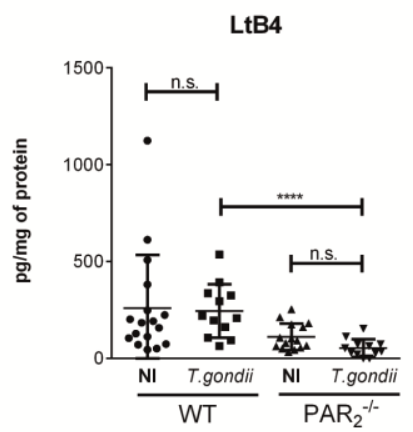

Figure 6. Quantification of lipid metabolites in the small intestine of WT and PAR 2 KO mice infected with Toxoplasma gondii.

This article is protected by copyright. All rights reserved. 
Figure 7 Bonnart et al.
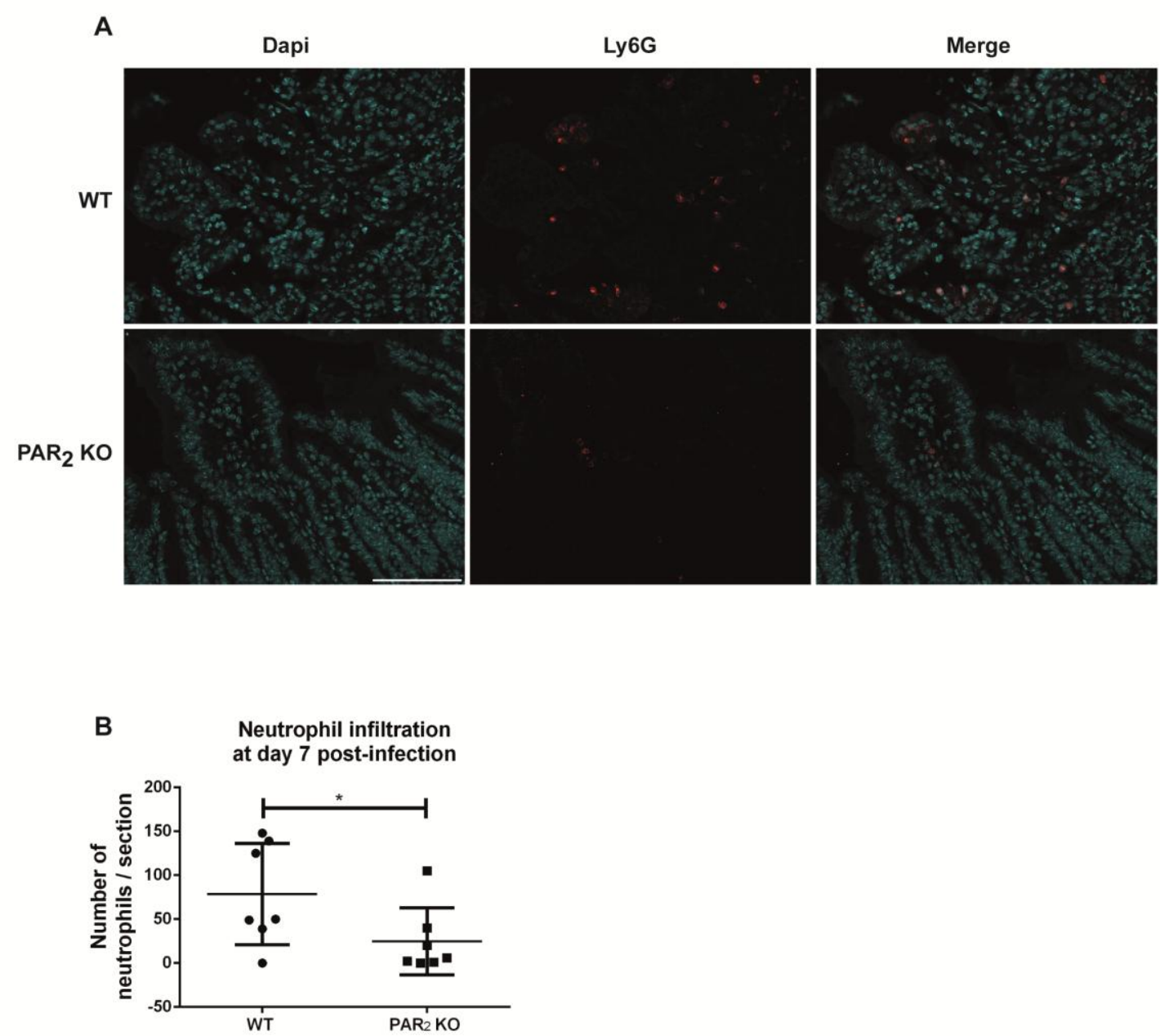

Figure 7. Neutrophil infiltration in the small intestine of infected WT and PAR 2 KO animals at day 7 post-infection.

This article is protected by copyright.All rights reserved.

Bonnart, C., Feuillet, G., Vasseur, V., Cénac, N., Vergnolle, N., Blanchard, N. (2017). 